\title{
A Dynamic Follow-Up of Pneumonia Caused by Coronavirus Disease 2019 (COVID-19) on CT Scan
}

\author{
Li Zhao ${ }^{1}$, Bo Chen ${ }^{1}$, Ya'nan Huang ${ }^{1}$, Minxia Yang ${ }^{1}$, Jianfeng Yang ${ }^{1}$ and Zhenhua Zhao ${ }^{1,{ }^{*}}$ \\ ${ }^{1}$ Department of Radiology, Shaoxing People's Hospital (the First Affiliated Hospital of Shaoxing University), Shaoxing, China \\ "Corresponding author: Department of Radiology, Shaoxing People's Hospital (The First Affiliated Hospital of Shaoxing University), No.: 568, Zhongxing North Road, Postal \\ Code: 312000, Shaoxing, China. Tel: +86-57588229281, Email: zha02075@163.com
}

Received 2020 March 18; Accepted 2020 April 21.

\begin{abstract}
Coronavirus disease (COVID-19) broke out in Wuhan, China. It is a highly contagious respiratory disease, the basic reproductive number (RO value) for COVID-19 has been reported as 2.68 with an epidemic doubling time of 6.4 days. In this case report, a 50-yearold man presented with a history of fever and chills. Chest radiographs failed to find any abnormality, and computed tomography (CT) showed ground glass shadow of the upper lobe of the left lung. Real-time polymerase chain reaction (RT-PCR) of the patient's pharyngeal swab was positive for COVID-19 nucleic acid. The course of the disease developed from mild to severe, then improved and recovered. The patient underwent antiviral drugs, low dose of glucocorticoids, symptomatic treatment, and immunoglobulin for human intravenous injection. We report this long time follow-up case of COVID-19.
\end{abstract}

Keywords: COVID-19, Pneumonia, Tomography

\section{Introduction}

In December 2019, coronavirus disease (COVID-19) broke out in Wuhan, China. It is a highly contagious respiratory disease, the basic reproductive number ( $\mathrm{RO}$ value) for COVID-19 has been reported as 2.68 with an epidemic doubling time of 6.4 days (1-4). High resolution CT (HRCT) is sensitive for lung lesion early detection to support initial diagnosis, as well as good at evaluating the lesion nature and locating the disease scope $(5,6)$. Here, we report one case of pneumonia caused by COVID-19. The dynamic changes we caught in the disease may better help us in understanding the whole process and providing promising suggestions for clinical decision making.

\section{Case Presentation}

A 50-year-old local man came back to Shaoxing form Wuhan, China on January 18, 2020. He visited the hospital on January 24, 2020 (counted as day 1) with fever, chills, and occasional cough, but no nasal discharge, myalgia, or diarrhea. On admission, the same day, his body temperature was $38^{\circ} \mathrm{C}$ and there were coarse breath sounds in both lungs at auscultation. Digital radiography (DR) of the chest failed to find any abnormality at that moment. Laboratory tests showed a normal total white blood cell count of $5.68 \times 10^{9} / \mathrm{L}$ (normal range, $4.0-10.0 \times 10^{9} / \mathrm{L}$ ), and the differential count showed 54.7\% neutrophils (normal range, $40.0 \%-75.0 \%$ ) and 30.9\% lymphocytes (normal range, $20.0 \%$ - 50.0\%). C-reactive protein was normal (2.44 mg/L; normal range, 0 - $4 \mathrm{mg} / \mathrm{L}$ ). Real-time polymerase chain reaction (RT-PCR) of the patient's pharyngeal swab was positive for COVID-19 nucleic acid the next day. Day 2, early CT lung manifestation was ground glass shadow of the upper lobe of the left lung on January 25 (Figure $1 \mathrm{~A}$ and $\mathrm{B}$ ), and local consolidation in the edge, with interlobular septal thickening. Pneumonia involved only one lobe of the lung. However, on day 9, his body temperature increased to $38.9^{\circ} \mathrm{C}$. Unenhanced chest CT illustrated multiple bilateral ground glass shadows (Figure $1 \mathrm{C}$ and $\mathrm{D}$ ), and local consolidation, without mediastinal lymphadenopathy or pleural fluid. Blood gas analysis: partial pressure of carbon dioxide was $31.4 \mathrm{mmHg}$, and partial pressure of oxygen was $66.7 \mathrm{mmHg}$. Meanwhile, the patient was treated with low dose of glucocorticoids and non-invasive ventilation. For the next 6 days, human immunoglobulin was injected intravenously to enhance the immunity of the patient. On day 15, after a period of support therapy, the patient's body temperature returned to normal; while, CT examination indicated that prior inflammation was absorbed slightly (Figure 1E and F).

Meanwhile, a small amount of pleural effusion on both 

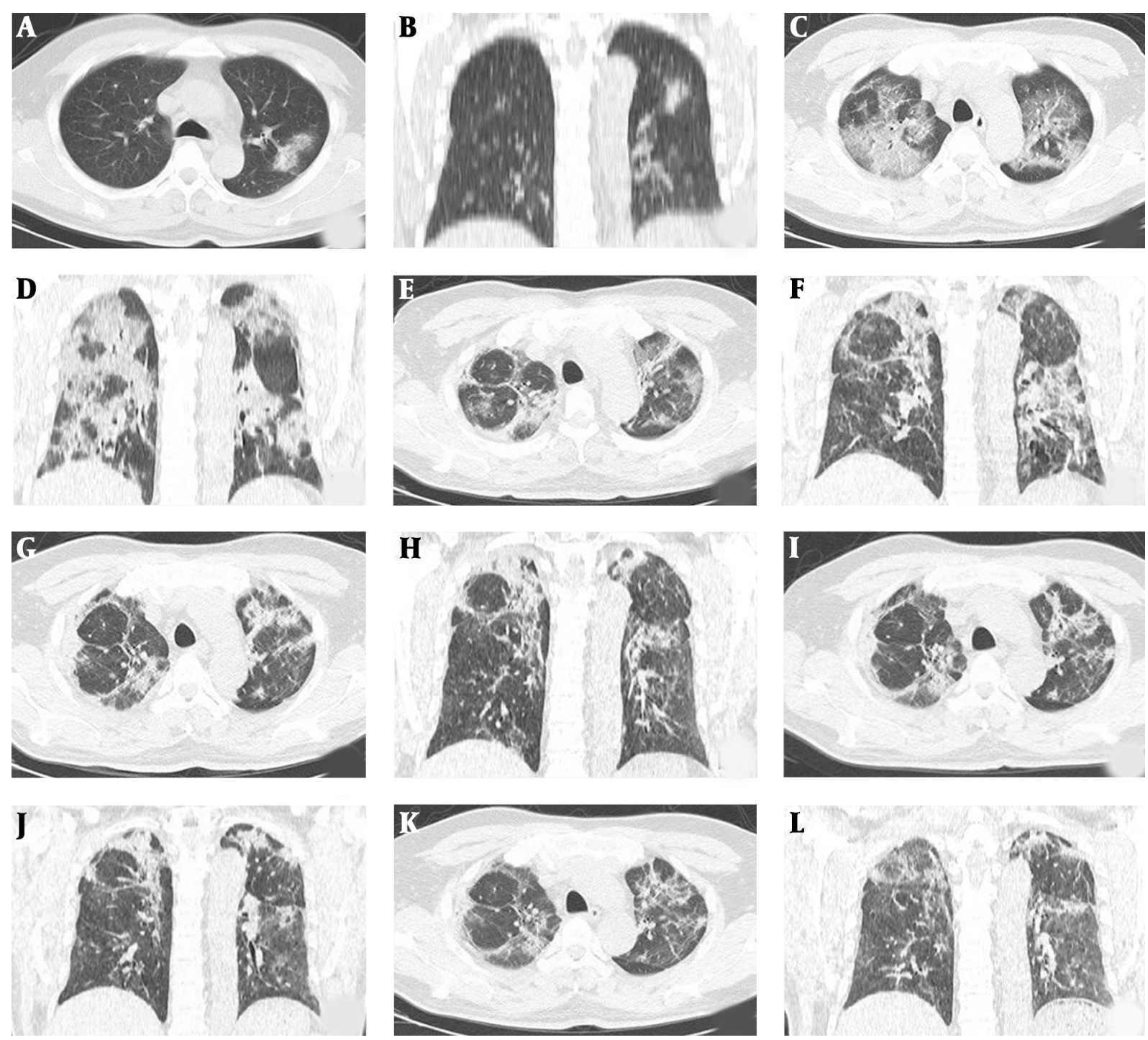

Figure 1. Unenhanced chest CT images of a 50-year-old man with COVID-19. A and B, Axial and coronal unenhanced chest CT showed ground glass shadow of upper lobe of left lung; $\mathrm{C}$ and D, As the disease progressed, unenhanced chest CT 9 days later showed extensive bilateral ground glass opacities and consolidation; $\mathrm{E}$ and F, After 15 days of therapy, the follow-up unenhanced chest CT showed that the consolidation area of the lung began to absorb; G and H, Unenhanced chest CT obtained 20 days later showed the consolidation area absorbed obviously; I and J, On day 25 after admission, the result of unenhanced chest CT showed the pneumonia was further absorbed; K and L, After 29 days of treatment, the disease improved, leaving the lung fiber cord focus.

sides was observed. Day 20, by the disease progressing, chest CT showed obvious absorbing of the ground glass shadow and the consolidating area of the lesion (Figure $1 \mathrm{G}$ and $\mathrm{H}$ ). On day 25 , pneumonia lesion was further absorbed, and the two lungs were scattered with fiber lesions (Figure $1 \mathrm{I}$ and J). No pleural effusion was detected anymore. On day 29, the lung showed fiber cord focus (Figure $1 \mathrm{~K}$ and L). Finally, on day 33, after using antiviral drugs, symptomatic treatment, and immunoglobulin for human intravenous injection, RT-PCR of the patient's pharyngeal swab was negative for COVID-19 nucleic acid. At the same time, we recorded the dynamic changes of laboratory parameters (Figure 2).

\section{Discussion}

It is believed that early CT scan is highly meaningful, which can target the lesion and reflect a coarse impression as early as possible. Meanwhile, the coronal reconstruction of the image is valuable to assess the therapeutic ef- 


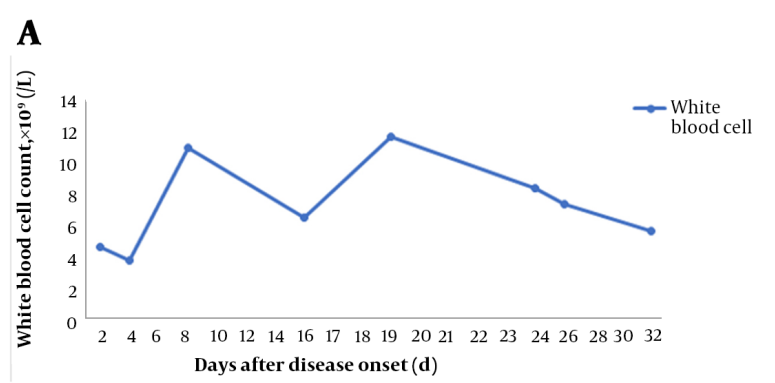

\section{B}
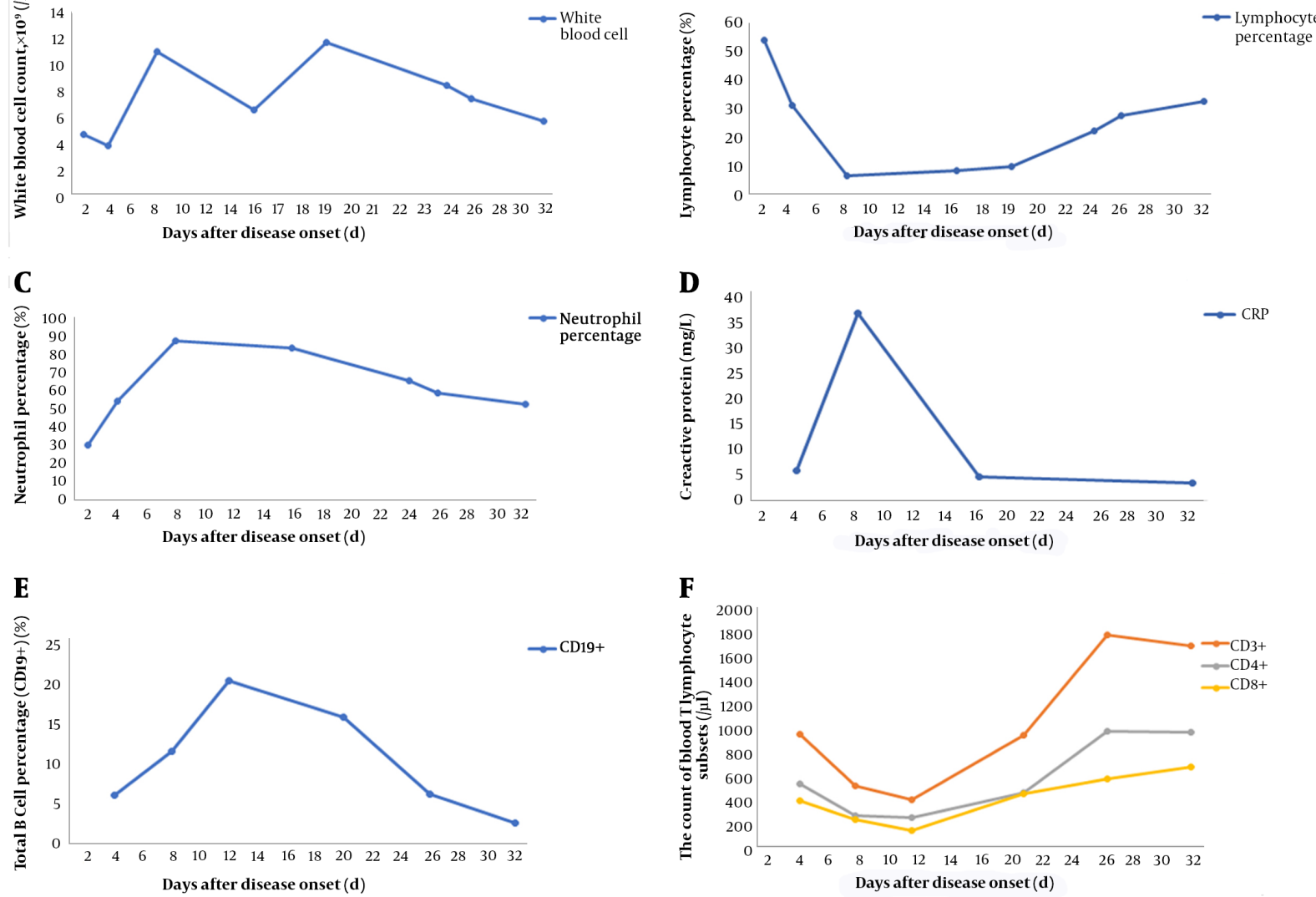

Figure 2. Dynamic profile of laboratory parameters in a patient with coronavirus disease (COVID-19). A, Dynamic changes of white blood cell count; B, Dynamic changes of lymphocyte percentage; C, Dynamic changes of neutrophil percentage; D, Dynamic changes of C-reactive protein; E, Dynamic changes of total B cell percentage (CD19 ${ }^{+}$); F, Dynamic changes of $\mathrm{T}$ lymphocyte subsets $\left(\mathrm{CD} 3^{+}, \mathrm{CD} 4^{+}, \mathrm{CD} 8^{+}\right)$.

fect through the illustration of the lesion shape and distribution, leading to an accurate clinical classification. As to the patient mentioned above, "white lungs" was observed at the second CT scan, with an interval of 7 days, revealing that the disease progressed quite rapidly. When the patients' manifestations are aggravating, CT scan should be carried out if possible, to follow up the lung lesions and it is usually checked every 2 days. On the other hand, if the etiology-confirmed patients are mild cases, a CT scan taken in an interval of 5- 7 days will be recommended to lower unnecessary radiation dose. In treatment, maintain proper innate immunity during anti-inflammation. Do not further activate the inflammatory response while improving the patient's immunity. It is necessary to strengthen immune support therapy. Immunoglobulin is recommended for the treatment of viral pneumonia.

\section{Footnotes}

Authors' Contributions: The first two authors contributed equally.

Conflict of Interests: None declared.

Ethical Approval: None declared.

Funding/Support: None declared.

Informed Consent: Informed consent forms were signed.

\section{References}

1. Yingxia L, Yang Y, Cong Z, Fengming H, Fuxiang W, Jing Y, et al. Clinical and biochemical indexes from 2019-nCoV infected patients linked to viral loads and lung injury. Sci China Life Sci. 2020. doi: 10.1007/s11427020-1643-8. [PubMed: 32048163].

2. Nanshan C, Min Z, Xuan D, Jieming Q, Fengyun G, Yang H, et al. Epidemiological and clinical characteristics of 99 cases of 2019 novel coronavirus pneumonia in Wuhan, China: a descriptive 
study. Lancet. 2020;395(10223):10.1016/S0140-6736(20)30211-7-513. doi: 10.1016/S0140-6736(20)30211-7. [PubMed: 32007143].

3. Joseph T, Kathy L, Gabriel M. Nowcasting and forecasting the potential domestic and international spread of the 2019-nCoV outbreak originating in Wuhan, China: a modelling study. Lancet. 2020. doi: 10.1016/S0140-6736(20)30260-9. [PubMed: 32014114].

4. Chaolin H, Yeming W, Xingwang L, Lili R, Jianping Z, Yi H, et al. Clinical features of patients infected with 2019 novel coronavirus in
Wuhan, China. Lancet. 2020;395(10223):10.1016/S0140-6736(20)301835-506. doi: 10.1016/S0140-6736(20)30183-5. [PubMed: 31986264].

5. Jeffrey P. Chest CT Findings in 2019 Novel Coronavirus (2019-nCoV) Infections from Wuhan, China: Key Points for the Radiologist. Radiology. 2020. doi: 10.1148/radiol.2020200241. [PubMed: 32017662].

6. Fengxiang S, Nannan S, Fei S, Zhiyong Z, Jie S, Hongzhou L, et al. Emerging Coronavirus 2019-nCoV Pneumonia. Radiology. 2020. doi: 10.1148/radiol.2020200274. [PubMed: 32027573]. 